\title{
A Rare Case of Right Upper Lung Cancer With Azygos Lobe and Partial Anomalous Pulmonary Venous Return: A Case Report
}

Xiaoyang SU ( $\nabla$ xiaoyang_su@126.com )

Maoming People's Hospital https://orcid.org/0000-0002-0572-2702

Qianzhun Huang

Maoming People's Hospital

Zhiqiang Luo

Maoming People's Hospital

Ning Fang

Maoming People's Hospital

Jian Huang

Maoming People's Hospital

\section{Case report}

Keywords: Lung cancer, Azygos lobe, Partial anomalous pulmonary venous return, Three-dimensional reconstruction

Posted Date: July 24th, 2021

DOl: https://doi.org/10.21203/rs.3.rs-727590/v1

License: (c) (i) This work is licensed under a Creative Commons Attribution 4.0 International License. Read Full License 


\section{Abstract}

Background: The azygos lobe (AL) combined with partial anomalous pulmonary venous return (PAPVR) is comparatively uncommon as well as in radical surgery for right lung cancer.

Case presentation: We herein present an extremely rare case of lung cancer coexisting with AL and asymptomatic PAPVC, which was diagnosed with preoperative contrast three-dimensional reconstruction and received radical surgery by thoracoscopy. During the surgery, we preserved azygos vein successfully and found a split type of PAPVR in right upper pulmonary vein.

Conclusions: AL combined with PAPVR may cause confusion on the vascular separation and disconnection of the right pulmonary hilar. However, preoperative 3D reconstruction are more conducive to the correct performing of this type of surgery.

\section{Background}

The azygos lobe (AL) is an rare variantion in the right upper lobe (RUL), resulting from abnormal migration of the azygos vein (AV) from the thoracic wall to its usual position at the tracheobronchial angle ${ }^{[1]}$. Partial anomalous pulmonary venous return (PAPVR) is a comparatively uncommon congenital pulmonary venous anomaly that involves drainage of 1 to 3 pulmonary veins into the right-sided circulation ${ }^{[2]}$. The prevalence of the AL is $1.2 \%$ in CT scans while PAPVR is only $0.1-0.2 \%{ }^{[3]}$. At present, there is no report on combination of such two type malformations as well as radical surgery for right lung cancer. Herein we present the case of lung cancer coexisting with AL and asymptomatic PAPVC, which was diagnosed with preoperative contrast three-dimensional reconstruction (3D- reconstruction) and received radical surgery. During the surgery, we preserved AV successfully to avoid massive hemorrhage and found a split type of PAPVR in right upper pulmonary vein (RUV), which is confused with right middle pulmonary vein (RMV). Therefore, we have summarized some previous experience regarding such vascular malformations.

\section{Case Presentation}

A 60-year-old male patient was admitted to our department due to incidental observation of a 10-mm irregular, ill-defined partly solid nodule in RUL by annual physical CT scans(Fig. 1A). The tumour markers were elevated and malignant nodule was highly suspected, squamous cell carcinoma antigen 1.70 $\mathrm{ng} / \mathrm{mL}$ (reference 0-1.5ng/mL), CYFRA21-1 3.99ng/ml (reference 0-2.1ng/mL). Pulmonary function tests were appropriate for lung resection; FVC 4.76L (Pred 131.4\%), FEV1 3.89 L (Pred 97.4\%) and the diffusion function (MVV) 164.97L/min (Pred 97.4\%) without significant abnormalities of echocardiography. Contrast CT described abnormal courses of AV and separated the AL from RULDFig. 1BQ. Therefore, a preoperative 3D-reconstruction was performed which revealed AL combined a split type of PAPVR in RUV.

The RUV was splited into two branches with different diameters(Fig. 2). The thicker branch drained most of the anterior and atypical veins as well as part of the posterior veins $(\mathrm{V} 1+\mathrm{V} 3 \mathrm{ac}+\mathrm{V} 2 \mathrm{~b})$, which was 
recognized as PAPVR, accompanying AV into the superior vena cava. While the other small branch (V2ac $+\mathrm{V} 3 \mathrm{~b}$, represented by sRUV below) drained into the left atrium at the proximal end of RMV.

After excluding distant metastasis, two-port thoracoscopic right upper lobectomy and mediastinal lymph node dissection were performed. During the surgery, the abnormally dilated AV connected to the superior vena cava, and crossed the apex of the lung from the ventral to the dorsal side(Fig. 3A). When the RUL pulled down to the caudal side, the AL could be slipped out from the cavity consist of AV and pleuras smoothly(Fig. 3B). In the hilar, the thicker RUV branch draining into the superior vena cava was confirmed(Fig. 3C). After sutured the PAPVR of RUV by stapler, the pulmonary artery and bronchi of RUL were exposed as normal. For the hypoplastic of the fissure between the middle and upper lobe, we carefully separate sRUV, the veins of middle and lower lobe on mediastinal side. Confirming the RMV safety, the sRUV was ligated with silk thread and the fissure was separated by stapler. Shaped like a clover, the resected RUL combined AL was consistent with the 3D- reconstruction(Fig. 3F).

The operation time was 285 minutes with $50 \mathrm{ml}$ blood loss. The postoperative course was uneventful. The drainage tube was removed on the 3th and the patient was discharged on the 10th postoperative day in good clinical condition. Pathological analysis showed lung adenocarcinoma without involvement lymph nodes.

\section{Discussion And Conclusion}

AL combined with PAPVC is a comparatively uncommon variation in RUL, which is usually diagnosed incidentally on imaging with asymptomatic. The AL results from a failure of the normal migration of the $A V$ from the thoracic wall to its usual position at the tracheobronchial angle, such that the fissure of the parietal and visceral pleura remain invaginated, thus forming a cavity with the AV at its base ${ }^{[1,4]}$. Mostly, the potential cavity would not cause adhesion to the AL ${ }^{[5]}$. PAPVR is often associated with hypoplastic right lung and congenital heart disease, such as atrial septal defect, resulting in a left-to-right shunt. The patient's clinical severity is determined by the degree of the left-to-right shunt ${ }^{[6]}$. Inadequate recognition of $\mathrm{AL}$ may easily injury AV leading to mass hemorrhage $\mathrm{e}^{[4]}$. Lobectomy was the definitive treatment for lung cancer and PAPVC, and no hemodynamic problems occurred during and after the operation ${ }^{[7]}$. However, if PAPVR is located in different lobe to be preserved, inadequate resection may increase the left-to-right shunt flow and cause right-sided heart failure, increasing the risk of postoperative mortality ${ }^{[8]}$.

In this case, AL was discovered accidentally with asymptomatic. To understand the variantions of mediastinal vascular, we performed preoperative 3D reconstruction. The split RUV was found unexpectedly and partially as PAPVR. However the patient had no abnormalities of preoperative echocardiography without symptomatic, for the small degree of the left-to-right shunt. Intraoperatively, we found that the azygos fissure did not cause adhesions of AL. Hypoplastic of the fissure between the middle and upper lobe, the thicker branche of RUV dentified PAPVR may be misjudged as intact RUV, while the split small branche (sRUV) was confused with RMV(Fig. 3E), resulting in incomplete resection of 
RUV. Moreover, in this case, the PAPVR was mainly located in the RUV and lobectomy did not increase the flow of left-to-right shunt.

To sum up, if discovering AL before surgery, it should be aware that there may be other vascular variations. Therefore, completing contrast $\mathrm{CT}$ and vascular reconstruction maybe a great option. The appearance of $A L$ would not increase the difficulty of lobectomy, and AV could be preserved successfully. As for such vascular malformations as PAPVR, echocardiography should be performed to exclude congenital heart disease. Being familiar with anatomy of PAPVR is good for preventing accidental damage to the blood vessel, and especially when split type of RUV combined with hypoplasia fissure. Of course, preoperative 3D reconstruction are more conducive to the correct performing of this type of surgery, such as the identification of small branch of RUV and preservation of RMV, which is both safe and fast.

\section{Abbreviations}

AL

Azygos lobe

AV

Azygos vein

PAPVR

Partial anomalous pulmonary venous return

RUL

Right upper lobe

RUV

Right upper pulmonary vein

RMV

Right middle pulmonary vein

\section{Declarations}

\section{Acknowledgments}

Not applicable.

\section{Authors' contributions}

(1) Information collection, analysis and writing: Xiaoyang Su. (2)Surgical operation: Zhiqiang Luo, Jian Huang. (3)Data collection: Qianzhun Huang. (4)Imaging interpretation: Ning Fang. (5)Final approval of manuscript: All authors.

\section{Funding}


This study was supported by the high-level hospital construction research project of Maoming People's Hospital.

\section{Availability of data and materials}

Not applicable.

\section{Ethics approval and consent to participate}

Not applicable.

\section{Consent for publication}

Written informed consent was obtained from the patient for publication of this case report.

\section{Competing interests}

All the authors declare that they have no conflict of interest.

\section{Author details}

${ }^{1}$ Department of Thoracic Surgery, Maoming People's Hospital, Maoming, 101 Weimin Road, 52500, China.

\section{References}

[1] Bostanci K, Ozyurtkan M O, Polat M O, Batirel H, Lacin T, Yuksel M, Stamenovic D. Variations in pulmonary fissural anatomy: a medicolegal autopsy study of 256 cases[J]. ANZ J Surg,2020,90(4):608611.

[2] Ho M L, Bhalla S, Bierhals A, Gutierrez F. MDCT of partial anomalous pulmonary venous return (PAPVR) in adults[J]. J Thorac Imaging,2009,24(2):89-95.

[3] Al-Mnayyis A A, Al-Alami Z, Altamimi N, Alawneh K Z, Aleshawi A. Azygos Lobe: Prevalence of an Anatomical Variant and Its Recognition among Postgraduate Physicians[J]. Diagnostics,2020,10(7):470.

[4] Kauffman P, Wolosker N, de Campos J R M, Yazbek G, Jatene F B. Azygos Lobe: A Difficulty in VideoAssisted Thoracic Sympathectomy[J]. The Annals of Thoracic Surgery,2010,89(6):e57-e59.

[5] Arai H, Inui K, Kano K, Nishii T, Kaneko T, Mano H, Sasaki T, Masuda M. Lung cancer associated with an azygos lobe successfully treated with video-assisted thoracoscopic surgery[J]. Asian $\mathrm{J}$ Endosc Surg,2012,5(2):96-99.

[6] Heineman D J, van den Aardweg J G, Schreurs W H. A rare case of anomalous venous drainage of the right upper lobe[J]. J Thorac Dis,2015,7(10):E502-E504. 
[7] Kawasaki H, Oshiro Y, Taira N, Furugen T, Ichi T, Yohena T, Kawabata T. Partial Anomalous Pulmonary Venous Connection Coexisting with Lung Cancer: A Case Report and Review of Relevant Cases from the Literature[J]. Annals of Thoracic and Cardiovascular Surgery,2017,23(1):31-35.

[8] Black M D, Shamji F M, Goldstein W, Sachs H J. Pulmonary resection and contralateral anomalous venous drainage: a lethal combination[J]. Ann Thorac Surg,1992,53(4):689-691.

\section{Figures}

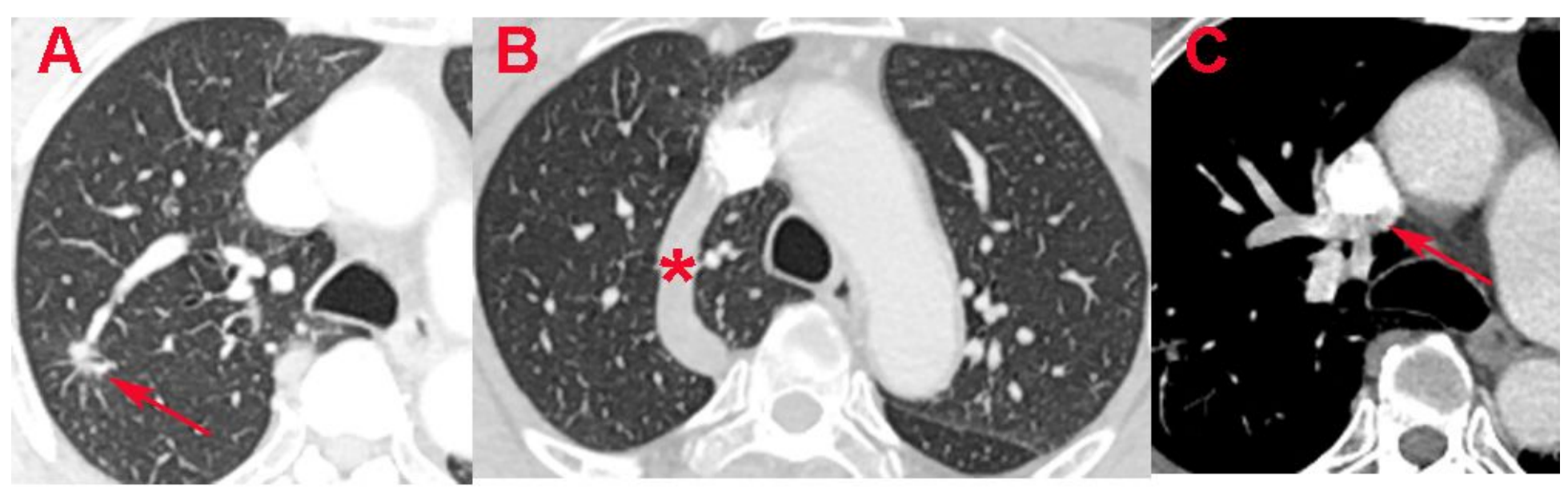

\section{Figure 1}

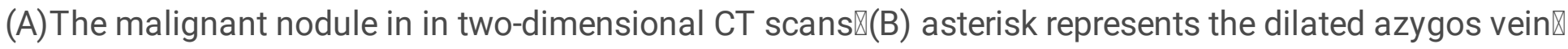
(C)The red arrow represents partial anomalous pulmonary venous return (PAPVR) drainages into superior vena cava.
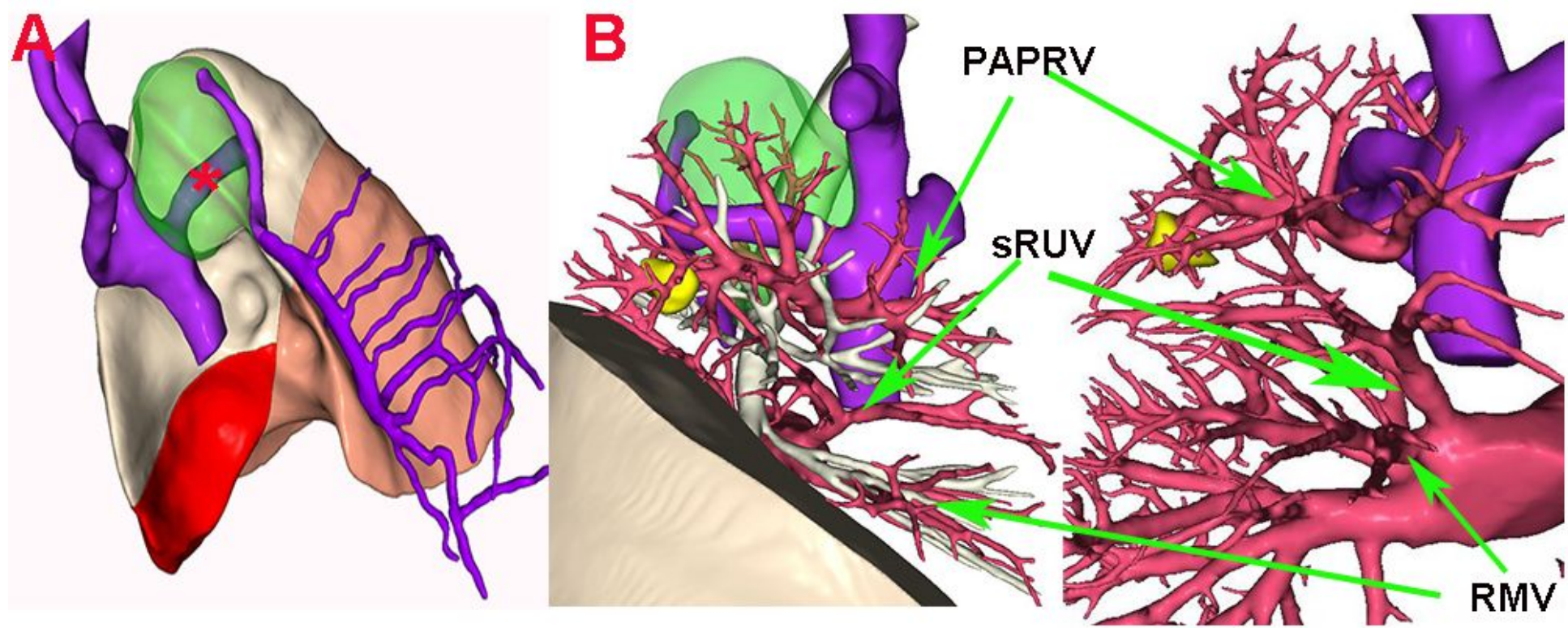


\section{Figure 2}

Three-dimensional reconstruction of computed tomography .(A) Asterisk represents the dilated azygos vein(AV), the green part of the lung is azygos lobe (AL). (B)PAPVR: partial anomalous pulmonary venous return; sRUV: small branch of right upper pulmonary vein; RMV:right middle pulmonary vein, and the nodule in yellow.
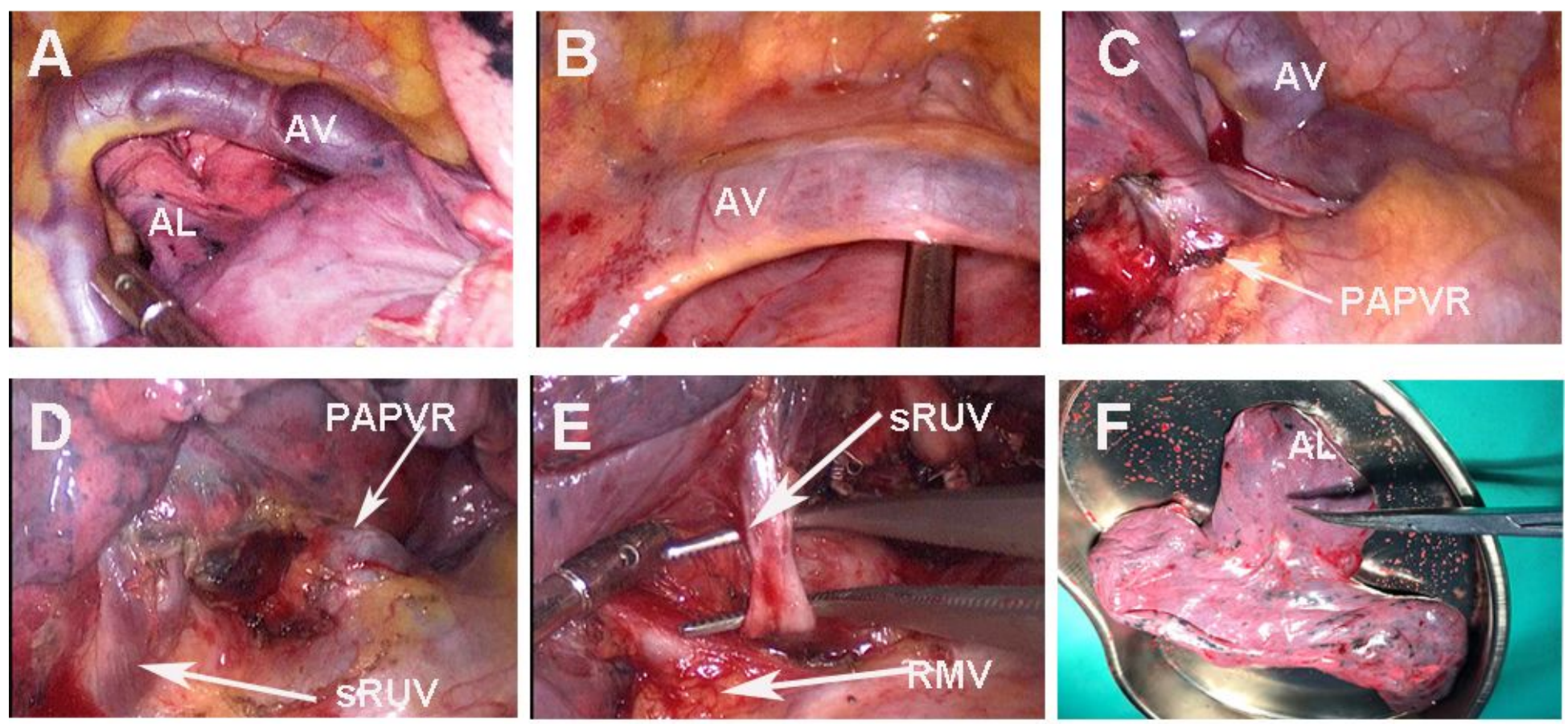

\section{Figure 3}

Intraoperative findings: (A) Dilated azygos vein (AV) and azygos lobe (AL), (B) The cavity formed by invaginated parietal and visceral pleura with AV. (C) Partial anomalous pulmonary venous return (PAPVR) and AV drainage into superior vena cava. (D and $E$ )The splited tppe of right upper pulmonary vein (RUV), sRUV: small branch of RUV, RMV:right middle pulmonary vein. (F) The resected right upper lobe combined AL shaped like a clove. 